\title{
PRODUÇÃO DO CONHECIMENTO SOBRE TEORIAS DE ENFERMAGEM: ANÁLISE DE PERIÓDICOS DA ÁREA, 1998-2007
}

Knowledge production on nursing theories: analysis of the area periodicals, 1998-2007

\author{
Produccion de conocimiento sobre teorias de enfermeria: \\ analisis de periodicos del area, 1998-2007
}

Diego Schaurich ${ }^{1}$

Maria da Graça Oliveira Crossetti

\begin{abstract}
RESUMO
Este estudo objetivou analisar a produção do conhecimento sobre teorias de enfermagem publicada em periódicos da área, entre 1998 e 2007. Trata-se de uma investigação descritiva, de natureza bibliográfica, com abordagem quantitativa. Foram encontrados e analisados 171 (4,6\%) estudos sobre teorias de enfermagem extraídos do total de 3.757 resumos publicados em sete periódicos científicos. A maioria destes artigos é oriunda de pesquisas, em que foram utilizadas a Teoria de Enfermagem Humanística, a Teoria do Cuidado Cultural e a Teoria do Autocuidado como principais referenciais em trabalhos publicados em dupla autoria e originados, predominantemente, nas regiões Sul e Nordeste do País. Acredita-se que esta investigação permitiu dar visibilidade a tal temática, bem como identificar algumas das características e tendências do conhecimento acumulado referente às teorias de enfermagem, na realidade brasileira.
\end{abstract}

Palavras-chave: Enfermagem. Produção Científica. Pesquisa em Enfermagem. Teoria de Enfermagem.

\begin{abstract}
A study of bibliographical nature approach which aimed to analyze the production of knowledge on nursing theories published in the area periodicals from 1997 to 2006. It was found and examined $171(4.6 \%)$ studies on theories of nursing from the total of 3,757 abstracts published in seven scientific journals. Most of the articles analyzed are from researches in which the Theory of Culture Care, the Humanistic Nursing Theory and Selfcare Theory were used as main references in works published in co-authorship and others from the same area, predominantly in the south and northeast regions of the country. It was a common belief that this investigation allowed a broader visibility to this theme as well as it identifies some characteristics and trends of gathered knowledge referred to the nursing theories in the Brazilian reality.
\end{abstract}

Keywords: Nursing. Scientific production. Nursing research. Nursing theory.

\section{Resumen}

Estudio de naturaleza bibliográfico que tuvo como objetivo el análisis de la producción de conocimiento sobre teorías de Enfermería, publicado en periódicos del área, entre 1997 y 2006. Se encontraron y se examinaron $171(4,6 \%)$ estudios sobre las teorías de la enfermería a partir de un total de 3.757 resúmenes publicados en siete revistas científicas. La mayoría de los artículos analizados tuvieron su origen en investigaciones en las cuales fueron utilizadas la Teoría del Cuidado Cultural, la Teoría de la Enfermería Humanística y la Teoría del Autocuidado como principales referenciales, en trabajos publicados en coautoría y que tuvieron origen, predominantemente, en las regiones Sur y Noreste del país. Se concluye que esta investigación permitió dar visibilidad a tal temática, así como identificar algunas de las características y tendencias del conocimiento acumulado en relación con las teorías de la Enfermería, en la realidad brasileña

Palabras Clave: Enfermería. Producción científica. Pesquisa en enfermería. Teoría de enfermería.

${ }^{1}$ Doutorando do Programa de Pós-Graduação da Escola de Enfermagem da Universidade Federal do Rio Grande do Sul (UFRGS/RS); Bolsista CAPES; Membro do Núcleo de Estudos do Cuidado em Enfermagem (NECE - UFRGS/RS); Membro do Grupo Cuidado às Pessoas, Famílias e Sociedade da Universidade Federal de Santa Maria (UFSM/RS). Brasil. E-mail: eu_diegosch@hotmail.com, ${ }^{2}$ Docente Adjunta do Departamento de Enfermagem Médico-Cirúrgica da Escola de Enfermagem da UFRGS. Doutora em Filosofia em Enfermagem pela Universidade Federal de Santa Catarina (UFSC/SC). Coordenadora do NECE (UFRGS). Brasil. E-mail: mgcrossetti@gmail.com 


\section{INTRODUÇÃO}

Ao longo do processo histórico, a Enfermagem vem se constituindo como ciência e arte na área da saúde com vistas a produzir um corpo de conhecimentos próprio que atenda aos interesses, necessidades e peculiaridades da profissão e do contexto social. 0 cuidado de enfermagem, para dar conta da complexidade e dinamicidade das questões que envolvem 0 estar saudável e o estar doente de indivíduos e/ou grupos populacionais, precisa abranger, além dos aspectos técnicocientíficos, os preceitos éticos, estéticos, filosóficos, humanísticos e culturais.

Entende-se que a Enfermagem só vem conseguindo consolidar-se como ciência e arte porque tem produzido uma linguagem específica que atribui significado aos elementos constitutivos do seu ser, saber e fazer. Assim, a linguagem produzida por determinado campo do saber possibilita a compreensão acerca das representações do pensamento e do mundo, seja como veículo de comunicação ou como instrumento de ação/interação. ${ }^{1}$

Portanto, se a pretensão é construir e promover a ciência da enfermagem, faz-se necessário tornar objetiva a sua linguagem, bem como "situar os termos que a constituem em um universo comum de percepção e de comunicação - o universo instituído pela cultura da enfermagem"1:231. Esta linguagem espećíica é representada, dentre outros elementos, pelas teorias de enfermagem que têm como objetivo maior definir, caracterizar e explicar/compreender/interpretar, a partir da seleção e interrelação conceitual, os fenômenos que configuram domínio de interesse da profissão $0^{1-2}$.

Para chegar à elaboração desta linguagem, a Enfermagem, durante muitos anos, descreveu seus procedimentos a partir da experiência da prática clínica para, nas primeiras décadas do século XX, sistematizá-los por meio das técnicas de enfermagem. Porém, foi somente nas décadas de 1950 e 1960 que a preocupação recaiu em refletir mais profundamente sobre a profissão, o seu objeto de trabalho e o referencial teórico pertinente ao mundo do cuidar, quando, então, começaram a ser elaboradas as teorias de enfermagem propriamente ditas. ${ }^{2}$

Compreende-se, desta forma, que as teorias de enfermagem "têm contribuído para a formação de uma base relativamente sólida de conhecimento, que organiza o mundo fenomenal da Enfermagem"1:231. Neste sentido, elas podem ser consideradas aportes epistemológicos fundamentais à construção do saber e à prática profissional, pois têm auxiliado na orientação dos modelos clínicos da enfermagem e têm possibilitado que os profissionais descrevam e expliquem aspectos da realidade assistencial, auxiliando no desenvolvimento da tríade teoria, pesquisa e prática na área.

As teorias de enfermagem foram elaboradas para explicitarem a complexidade e multiplicidade dos fenômenos presentes no campo da saúde e, também, para servirem como referencial teórico/metodológico/prático aos enfermeiros que se dedicam à construção de conhecimentos, ao desenvolvimento de investigações e à assistência no âmbito da profissão. Para tanto, estas teorias, de uma maneira geral, se estruturam a partir de quatro conceitos centrais, quais sejam: ser humano, saúde, meio ambiente (físico, social e simbólico) e enfermagem. Isto porque se faz relevante considerar que o conhecimento produzido a partir desta linguagem específica precisa levar em conta que o compromisso social da profissão está em aliar o cuidado de enfermagem às vivências e experiências de saúde humana. $^{3}$

Optou-se, então, por estudar a produção do conhecimento relativo às teorias de enfermagem publicada em periódicos da área, com o intuito de alcançar o estado da arte desta temática na realidade brasileira. Justifica-se a realização deste estudo por se considerar que as teorias de enfermagem são referenciais fundamentais à matriz disciplinar da profissão, pela relevância do tema para as áreas do ensino, pesquisa e assistência e, ainda, por possibilitar que sejam reveladas as nuances e tendências do conhecimento acumulado por esta área do saber. Tem-se por objetivo analisar a produção do conhecimento de teorias de enfermagem publicada em periódicos da área entre os anos de 1998 e 2007.

\section{PERCURSO METODOLÓGICO}

Trata-se de uma investigação descritiva, de natureza bibliográfica, com abordagem quantitativa. 0 estudo descritivo tem por finalidade permitir um aprofundamento do pesquisador em relação a determinado problema, visando a correlação dos fatos. A abordagem quantitativa foi eleita por propiciar a caracterização e quantificação dos objetivos propostos por meio de medidas apresentadas a partir de análises estatísticas simples. ${ }^{4}$

Ocorreu, então, a consulta a sete periódicos de enfermagem referentes ao período de 1998 a 2007. Como justificativas à escolha destes periódicos, podem-se citar os fatos de serem importantes veículos de disseminação e divulgação dos estudos publicados pela Enfermagem; estarem classificados com Qualis B e C Internacional pela Coordenação de Aperfeiçoamento de Pessoal de Nível Superior (CAPES) ${ }^{5}$; terem tido periodicidade regular e estarem disponíveis para consulta durante a pesquisa.

A coleta dos artigos foi feita junto à Biblioteca da Escola de Enfermagem da UFRGS e por meio dos arquivos dos exemplares acessados diretamente nos sites das revistas que os têm à disposição Esta busca foi realizada pelos pesquisadores durante os meses de janeiro e março de 2008, período considerado suficiente por possibilitar uma investigação criteriosa nos exemplares. Para identificar os estudos, procedeu-se à leitura de todos os resumos dos artigos publicados e, quando ocorriam dúvidas, realizava-se a leitura do texto na íntegra. Assim, a partir da seleção daqueles relativos às teorias de enfermagem, deu-se início à fase de análise dos estudos, que ocorreu entre os meses de abril e junho de 2008. Os dados foram tabulados e a análise quantitativa se deu por meio de frequência absoluta e relativa. 
Com a finalidade de organizar e sistematizar a análise dos artigos, elaborou-se um instrumento de avaliação dos estudos, constituído da seguinte maneira: local para identificação do trabalho (título, ano, volume, número e periódico de publicação) e espaço para a descrição do objetivo do estudo, da classificação

\section{APRESENTAÇÃO E DISCUSSÃO DOS RESULTADOS}

Considerando o período de 1998 a 2007 em que foram analisados estes sete periódicos da área de Enfermagem, ocorreu a leitura de um total de 3.757 resumos de artigos. Deste conjunto, foram localizados e incluídos 171 artigos que do artigo (reflexão, pesquisa e relato de experiência), da identificação da teoria de enfermagem utilizada, do número de autores, da titulação postulada pelo autor e da região geográfica de origem do estudo. Estes dados coletados configuraram as variáveis estudadas.

Tabela 1: Distribuição do total de artigos publicados/ano e do total de artigos referentes às teorias de enfermagem. Brasil. 1998-2007

\begin{tabular}{|cccc}
\hline Ano & Total de Artigo/ano & $\begin{array}{c}\text { Total de Artigos de } \\
\text { Teorias de } \\
\text { Enfermagem }\end{array}$ & $\mathrm{f}(\%)$ \\
\hline 1998 & 291 & 05 & 1,7 \\
1999 & 332 & 18 & 5,4 \\
\hline 2000 & 369 & 33 & 8,9 \\
\hline 2001 & 301 & 09 & 3,0 \\
2002 & 340 & 16 & 4,7 \\
2003 & 435 & 10 & 2,3 \\
\hline 2004 & 514 & 19 & 3,7 \\
2005 & 509 & 20 & 3,9 \\
\hline 2006 & 576 & 26 & 4,5 \\
\hline 2007 & 658 & 15 & 2,3 \\
Total & 3.757 & 171 & 4,6 \\
\hline
\end{tabular}

A análise da Tabela 1 evidencia que a utilização de teorias de enfermagem pelos autores brasileiros é irregular ao longo do período considerado para esta investigação. Entretanto, chama a atenção a quantidade de artigos publicados no ano de 2000 ( $n=33$ ), e observa-se que a produção relacionada a este conhecimento vem crescendo a partir de 2002. Quando se considera um biênio, têm-se os seguintes resultados: período de 2002 a 2003, 26 artigos; período de 2004 a 2005, 39 artigos; e, período de 2006 a 2007, 41 artigos; ou seja, constata-se um aumento na utilização e publicação de estudos subsidiados por estes referenciais específicos.

Esta constatação de maior emprego das teorias de enfermagem a partir de 2002 vem ao encontro de uma discussão um pouco mais antiga na profissão acerca da necessidade de conhecer/analisar as práticas de enfermagem e aliá-las à construção teórica para, assim, possibilitar a produção de novos conhecimentos e, consequentemente, a consolidação da Enfermagem como ciência e arte na área da saúde. Acredita-se que uma maior compreensão destes referenciais conceituais, bem como de sua aplicação na prática clínica e no desenvolvimento de pesquisas, propicie a elaboração de uma linguagem própria da Enfermagem.

A produção do conhecimento sobre teorias de enfermagem representa $4,6 \%$ do total de artigos publicados nestes periódicos. Embora possa representar uma frequência utilizaram teorias de enfermagem, o que representa 4,6\% do total de trabalhos publicados. Observa-se na Tabela $1 \mathrm{a}$ distribuição anual do total de artigos publicados nestes periódicos e dos artigos referentes às teorias de enfermagem. relativamente baixa, há que se considerar a tendência atual da Enfermagem brasileira em diversificar o referencial teórico e/ ou metodológico usado em seus estudos. Isso porque os enfermeiros vêm buscando a contribuição de outras áreas do conhecimento para dar conta da complexidade dos fenômenos que fazem parte de sua prática, conforme apontam outros estudos. $^{6-7}$

$\mathrm{Na}$ análise por periódico, constatou-se que a Revista Texto \& Contexto Enfermagem publicou 662 artigos, sendo 56 (8,4\%) relativos a teorias de enfermagem; a Revista Brasileira de Enfermagem publicou 931 artigos, sendo 41 (4,4\%) inclusos neste estudo; a Revista Latino-Americana de Enfermagem publicou 1.018 artigos, sendo 29 (2,8\%) referentes a teorias de enfermagem; a Revista Gaúcha de Enfermagem publicou 346 artigos, dos quais 14 (4\%) utilizaram esta temática; a Revista Acta Paulista de Enfermagem publicou 468 artigos, 13 dos quais referentes a teorias de enfermagem (2,8\%); a Revista da Escola de Enfermagem da USP publicou 564 artigos sendo $10(1,8 \%)$ inclusos neste estudo; e a Revista Mineira de Enfermagem publicou 336 artigos, 8 (2,4\%) dos quais sobre teorias de enfermagem.

Estes achados evidenciam que a Revista Texto \& Contexto Enfermagem foi o periódico que mais publicou estudos que utilizaram as teorias como aporte teórico/metodológico, destacando-se com 32,7\% do total. Isto se deve, em parte, por 
estar vinculada a um Programa de Pós-Graduação Stricto Sensu que apresenta grupos e linhas de pesquisa que incentivam a construção de estudos amparados nas teorias de enfermagem. Em segundo e terceiro lugares encontram-se, respectivamente, a Revista Brasileira de Enfermagem (24\%) e a Revista LatinoAmericana de Enfermagem (17\%), as quais também se caracterizam como importantes veículos de disseminação das produções brasileiras.

Constatou-se que 107 artigos (62,6\%) são oriundos de pesquisas, 50 (29,2\%) são reflexões e 14 (8,2\%) são relatos de experiência. Este dado revela que as teorias de enfermagem têm sido utilizadas como referencial teórico, como percurso metodológico e/ou como fundamentação conceitual para a análise das pesquisas na área, o que é de fundamental importância por propiciar a elaboração de novos saberes voltados às especificidades e peculiaridades do contexto prático da profissão. Percebe-se, ainda, que a Enfermagem brasileira vem intensificando esforços no desenvolvimento e publicação de investigações ${ }^{8}$ para, com isso, constituir seu corpus de conhecimento e buscar melhorias à saúde da população.

A identificação dos referenciais teóricos revelou que a Teoria de Enfermagem Humanística de Paterson e Zderad, a Teoria do Cuidado Cultural de Leininger e a Teoria do Autocuidado de Orem configuram os modelos conceituais mais pesquisados pela Enfermagem brasileira, conforme pode ser observado na Tabela 2.

Tabela 2: Distribuição dos artigos de acordo com a teoria de enfermagem utilizada. Brasil. 1998-2007.

\begin{tabular}{lcc}
\hline Teorias de Enfermagem & $\mathrm{n}$ & $\mathrm{f}(\%)$ \\
\hline Teoria de Enfermagem Humanistica de Paterson e Zderad & 29 & 17 \\
Teoria do Cuidado Cultural de Leininger & 27 & 15,8 \\
Teoria do Autocuidado de Orem & 23 & 13,5 \\
Teoria do Alcance de Metas de King & 17 & 9,9 \\
Teoria do Cuidado Transpessaal de Watson & 15 & 8,8 \\
Teoria das Necessidades Humanas Básicas de Horta & 11 & 6,4 \\
Teoria de Adaptação de Roy & 11 & 6,4 \\
Teoria da Relação Interpessoal de Travelbee & 09 & 5,3 \\
Teoria de Florence Nightingale & 06 & 3,5 \\
Outras* & 04 & 2,3 \\
Teoria das Relações Interpessoais de Peplau & 04 & 2,3 \\
Teoria do Tornar-se Humano de Parse & 04 & 2,3 \\
\hline Várias teorias & 06 & 3,5 \\
Teoria do Modelo Conceitual de Rogers & 03 & 1,8 \\
Teoria do Cuidado Ético de Benner & 02 & 1,2 \\
\hline Total & 171 & 100 \\
\hline
\end{tabular}

*Teorias de Neuman, Levine, Orlando e Pender

A Teoria de Enfermagem Humanística de Paterson eZderad representa o referencial mais utilizado nos estudos (17\%), 0 que vem ao encontro dos achados de outras investigações. ${ }^{7,9-10}$ Isso demonstra a necessidade, atualmente vivenciada, de resgatar a dimensão humanística do cuidado, fenômeno que vem se processando na profissão. Isso porque este referencial, por possuir fundamentação na epistemologia existencialfenomenológica, permite lançar um olhar ao homem como ser que vivencia e experiencia situações existenciais únicas de saúde e de doença e, assim, servir como contraponto ao modelo biomédico que ainda impera na área da saúde. ${ }^{11}$

A Teoria do Cuidado Cultural de Leininger foi utilizada em $15,8 \%$ dos estudos e também desponta como importante referencial identificado em outros estudos. ${ }^{7,10}$ Esta teoria vem sendo empregada pela Enfermagem brasileira por permitir a compreensão de que os fenômenos de saúde e doença que permeiam o vivido de indivíduos e/ou grupos populacionais apresenta relação com os hábitos cotidianos, as crenças, os costumes e demais aspectos que configuram suas culturas, pois o cuidado é culturalmente definido. ${ }^{12}$ Além disso, pode-se destacar o fato de que este referencial apresenta uma proposta metodológica própria, o que favorece o desenvolvimento de pesquisas.

A Teoria do Autocuidado de Orem esteve presente em $13,5 \%$ do total analisado. Acredita-se que a escolha por esta teoria se deva por dois principais motivos: pelo fato de este referencial possibilitar, a partir de uma ação também educativa do enfermeiro, o desenvolvimento de atitudes que facultem aos indivíduos e/ou grupos populacionais o autocuidado, e devido ao avanço das condições crônicas de doença que têm 
exigido dos indivíduos a utilização a longo prazo de tratamento, o uso de tecnologias no domicílio e a reestruturação de estilos de vida, como formas de cuidado de si.

Estas três teorias de enfermagem juntas respondem por $46,3 \%$ do total analisado e podem ser consideradas importantes referenciais para a fundamentação da teoria e prática profissional. Isso porque, de uma maneira geral, percebe-se que elas apresentam olhares voltados para as vivências e experiências individuais e coletivas que estão envolvidas nos fenômenos de saúde e de doença, além de estimularem a produção de saberes que apontem alternativas e/ou soluções às dificuldades das práticas de enfermagem e a superação do modelo que orienta e organiza as instituições de saúde.
Ao que tange à quantidade de autores por artigo, foram obtidos os seguintes resultados: 50,9\% dos artigos tinham dupla autoria; $25,8 \%$, três autores; $15,2 \%$ foram publicados sem parceria; 5,8\% tinham a participação de quatro autores; $e$, 2,3\% foram publicados com a presença de mais de cinco autores. A publicação de estudos com a presença de dois ou mais autores ocorreu em $84,8 \%$ do total analisado, e estas parcerias vêm sendo, cada vez mais, uma exigência da CAPES e dos Programas de Pós-Graduação Stricto Sensu, assim como um resultado dos projetos e trabalhos desenvolvidos pelos grupos de pesquisa. Percebe-se que os achados deste estudo convergem com 0 encontrado em outras investigações, ${ }^{8,13}$ demonstrando ser uma tendência da Enfermagem brasileira.

Tabela 3: Distribuição dos autores dos artigos de teorias de enfermagem quanto ao grau de titulação postulado. Brasil. 1998-2007.

\begin{tabular}{lcc}
\hline Grau de Titulação dos Autores & $\mathrm{n}$ & $\mathrm{f}(\%)$ \\
\hline Doutor & 189 & 47,7 \\
Mestre & 69 & 17,4 \\
Estudante de Doutorado & 53 & 13,4 \\
Estudante de Mestrado & 45 & 11,4 \\
Especialista & 12 & 3,0 \\
Não Informado & 12 & 3,0 \\
Graduando(a) & 10 & 2,5 \\
Graduado(a) & 04 & 1,0 \\
Estudante de Especialização & 01 & 0,3 \\
Estudante de Pós-Doutorado & 01 & 0,3 \\
\hline Total & 396 & 100 \\
\hline
\end{tabular}

Quanto à titulação informada pelos autores (Tabela 3), constata-se a predominância de doutores $(47,7 \%)$, de estudantes de pós-graduação - mestrado e doutorado $(24,8 \%)$ e de mestres (17,4\%), revelando que "quem produz são os atores envolvidos na formação stricto sensu, daí a predominância de trabalho no gênero pesquisa" 8:648 Estes dados, aliados às parcerias intelectuais que se formam para a publicação, corroboram com a compreensão de que, também em relação à produção referente a teorias de enfermagem, os Grupos de
Pesquisa e os Programas de Pós-Graduação estão se consolidando como importantes produtores de conhecimento.

Em relação à região geográfica em que os estudos se originaram, dividiu-se a análise de acordo com o estado e a região brasileira. Os estados que mais publicaram artigos relacionados a teorias de enfermagem foram: Ceará, com 49 artigos (26,5\%); Rio Grande do Sul, com 34 artigos (21,8\%); Santa Catarina, com 30 artigos (17,9\%); e São Paulo, com 14 artigos $(8,6 \%)$. Quanto às regiões brasileiras, pode-se observar a Tabela 4.

Tabela 4: Distribuição dos artigos de teorias de enfermagem quanto à região geográfica em que o estudo se originou. Brasil. 1998-2007.

\begin{tabular}{lcc}
\hline Regiões Geográficas & $\mathrm{N}$ & $\mathrm{f}(\%)$ \\
\hline Região Sul & 77 & 45,1 \\
Região Nordeste & 55 & 32,2 \\
Região Sudeste & 30 & 17,5 \\
Região Centro-Oeste & 04 & 2,3 \\
Exterior* & 05 & 2,9 \\
\hline Total & 171 & 100 \\
\hline
\end{tabular}

*Venezuela (2), Espanha, Canadá e EUA. 
Quando a análise recai sobre os estados, percebe-se que os que mais pesquisaram sobre teorias de enfermagem foram 0 Ceará ( $n=49$ ) e o Rio Grande do Sul ( $n=34)$, o que pode ser explicado, em partes, pelo fato de que há grupos de estudos e linhas de pesquisas que se interessam em explorar e desenvolver esta temática. Há de se considerar, entretanto, que os estados de Santa Catarina e de São Paulo também apresentam grupos consolidados no estudo de teorias de enfermagem e que o motivo de não se configurarem como os primeiros desta lista pode estar relacionado ao fato de terem publicado seus trabalhos em outros periódicos não analisados.

Outro estudo ${ }^{9}$ que analisou a produção científica elaborada pela Pós-Graduação em Enfermagem da Universidade Federal do Ceará no período de 1993 a 2002, especificamente no que tange à área da saúde da mulher, revelou que $66,7 \%$ das dissertações e teses produzidas utilizaram teorias de enfermagem. Sendo assim, entende-se a liderança deste estado no emprego de referenciais conceituais da profissão, uma vez que a grande maioria destes estudos culmina como publicações em periódicos de abrangência nacional/internacional.

Contudo, ao analisar a região geográfica brasileira origem dos estudos desenvolvidos, tem-se que a região Sul foi a que mais contribuiu $(45,1 \%)$ com a construção do conhecimento envolvendo teorias de enfermagem, seguida pela região Nordeste, com $32,2 \%$ do total analisado, provavelmente pelos motivos anteriormente ressaltados. Outras investigações ${ }^{14-16}$ que analisaram o conhecimento produzido pela Enfermagem brasileira em relação a outras temáticas espećíicas da profissão apontam o Sudeste como a região que mais investe na elaboração e publicação de estudos, o que vem de encontro com os achados desta pesquisa, que constatou que as regiões Sul e Nordeste são os principais locus dos estudos sobre teorias de enfermagem.

\section{CONSIDERAÇÕES FINAIS}

As teorias de enfermagem representam um dos elementos que compõem a linguagem específica elaborada pelos atores profissionais, objetivando consolidar a Enfermagem como ciência e arte na área da saúde. Compreende-se que o emprego destes modelos conceituais, seja por meio de investigações, reflexões ou relatos de aplicação prática, possibilita perceber a valorização conferida pelos enfermeiros em relação a este conhecimento, bem como vislumbrar que esta temática vem sendo estudada pelo cenário acadêmico, tanto a nível de graduação como de pós-graduação.

Este estudo analisou a produção do conhecimento de teorias de enfermagem na realidade brasileira, o que possibilitou entender como evoluiu parte importante do saber construído pela profissão. A análise revelou alguns aspectos dos estudos publicados que se referem aos modelos teóricos da Enfermagem, permitindo compreender certas nuances desta temática nos cenários do ensino, da pesquisa, da extensão e da prática profissional.
Entre estas características, destaca-se que o conhecimento elaborado relativo às teorias de enfermagem vem apresentando um aumento a partir do biênio 2002-2003. Os trabalhos são predominantemente oriundos de pesquisas e têm utilizado a Teoria de Enfermagem Humanística (Paterson e Zderad), a Teoria do Cuidado Cultural (Leininger) e a Teoria do Autocuidado (Orem) como os principais referenciais teóricos empregados nos estudos. Ainda, observa-se que estes artigos são escritos, principalmente, em dupla parceria e por autores com a titulação de doutor, em especial pertencentes às regiões Sul e Nordeste do Brasil.

Acredita-se, portanto, que o "uso da teoria de enfermagem apoia os enfermeiros na definição de seus papéis, na aproximação da realidade e conseqüente adequação e qualidade do desempenho profissional, bem como na produção de conhecimento":66 e de uma linguagem própria. Por fim, considera-se que esta investigação permitiu dar visibilidade aos estudos que vêm se desenvolvendo vinculados às teorias de enfermagem e, também, em relação às principais características e tendências identificadas a partir da análise do conhecimento acumulado sobre tal temática, com vistas a contribuir com as áreas do ensino, da pesquisa e da prática assistencial da Enfermagem.

\section{REFERÊNCIAS}

1 Garcia TR, Nóbrega MML. Contribuição das teorias de enfermagem para a construção do conhecimento da área. Rev Bras Enferm 2004 mar/abr; 57(2): 228-32.

2 Souza MF. Teorias de enfermagem: importância para a profissão. Acta Paul Enferm 1988 set; 1(3): 63-5.

3 Thofehrn MB, Leopardi MT. Teorias de enfermagem, trabalho e conhecimento contemporâneo. Texto\&Contexto Enferm 2002 jan/abr; 11(1): 86-104.

4 Polit DF, Beck CT, Hungler BP. Fundamentos de pesquisa em enfermagem. $5^{\text {a }}$ ed. Porto Alegre(RS): Artmed; 2004.

5 Coordenação de Aperfeiçoamento de Pessoal de Nível Superior CAPES. Qualis CAPES-Periódicos de Enfermagem 2007. [ citado 18 maio 2008]. Disponível em: http://www.capes.gov.br.

6 Marques SC, Tyrrel MAR, Oliveira DC. A produção científica da enfermagem na perspectiva da representação social. Brasil, 19752001. Rev Latino-am Enfermagem 2006 set/out; 14(5): 762-69.

7 Erdmann AL, Leite JL, Mendes IAC, Trevizan MA, Dantas CC. Análisis de investigaciones brasileñas enfocadas en el cuidado de enfermería, años 2001-2003. Cienc Enferm 2005 jul/dez; XI(2): 35-46.

8 Rodrigues RM, Bagnato MHS. Pesquisa em enfermagem no Brasil: problematizando a produção de conhecimentos. Rev Bras Enferm 2002 nov/dez; 56(6): 646-50.

9 Moura ERF, Franco ES, Fraga MNO, Damasceno MMC. Produção científica em saúde da mulher na Pós-Graduação em Enfermagem da Universidade Federal do Ceará, Brasil 1993-2002. Cienc Enferm 2005 $\mathrm{jul} / \mathrm{dez}$ XI(2); 59-70. 
10 Marcon SS, Waidman MAP, Decesaro MN, Arêas MMM. Produzindo conhecimento sobre família: a contribuição da enfermagem do sul do Brasil. Acta Paul Enferm 2006 jan; 19(1): 21-7.

11 Paula CC, Schaurich D, Padoin SMM, Crossetti MGO. 0 cuidado como encontro vivido e dialogado na Teoria de Enfermagem Humanística de Paterson e Zderad. Acta Paul Enferm 2004 out/dez; 17(4): 425-31.

12 Moura MAV, Chamilco RASI, Silva LR. A teoria transcultural e sua aplicação em algumas pesquisas de enfermagem: uma reflexão. Esc Anna Nery Rev Enferm. 2005 dez; 9(3): 343-40.

13 Matheus MCC, Adami NP, Botta LMMG, Silva CV, Castro RAP. Perfil da Acta Paulista de Enfermagem em uma década de existência. Acta Paul Enferm 1998 dez; 11 (n. esp.): 7-13.
14 Erdmann AL, Silva IA, Rodrigues RAP, Fernandes JD, Vianna LAC, Lopes MJM, et al. Teses produzidas nos Programas de Pós-graduação em Enfermagem de 1983 a 2001. Rev Esc Enferm USP 2005 dez; 39(n. esp): 497-505.

15 Godoy MTH, Munari DB. Análise da produção científica sobre a utilização de atividades grupais no trabalho do enfermeiro no Brasil: 1980 a 2003. Rev Latino-am Enfermagem 2006 set/out; 14(5): 786-93.

16 Oliveira DC, Costa TL, Gomes AMT, Acioli S, Formozo GA, Heringer A, et al. Análise da produção de conhecimento sobre o HIV/AIDS em resumos de artigos em periódicos brasileiros de enfermagem, no período de 1980 a 2005. Texto\& Contexto Enferm 2006 out/dez; 15(4): 654-62. 